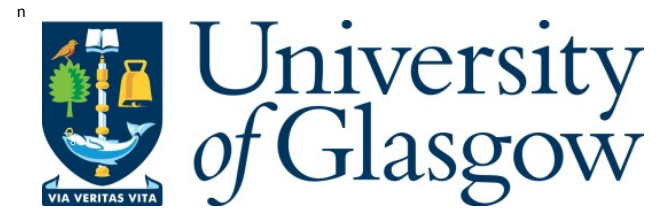

Cristiano, R., Ejrnaes, M., Casaburi, A ., Zen, N ., and Ohkubo, M . (2015) Superconducting nano-strip particle detectors. Superconductor Science and Technology, 28(12), 124004.

There may be differences between this version and the published version. $Y$ ou are advised to consult the publisher's version if you wish to cite from it.

http://eprints.gla.ac.uk/113878/

Deposited on: 22 J anuary 2016

Enlighten - Research publications by members of the U niversity of Glasgow http://eprints.gla.ac.uk 


\title{
Superconducting nano-strip particle detectors
}

\author{
R. Cristiano ${ }^{1}$, M. Ejrnaes ${ }^{1}$, A. Casaburi ${ }^{2}$, N. Zen ${ }^{3}$, M. Ohkubo ${ }^{4}$ \\ ${ }^{1}$ CNR - SPIN Institute of Superconductors, Innovative Materials and Devices, UOS-Napoli, I- \\ 80125 Napoli, Italy \\ ${ }^{2}$ School of Engineering, University of Glasgow, Glasgow G12 8LT, United Kingdom \\ ${ }^{3}$ National Institute of Advanced Industrial Science and Technology (AIST), Nanoelectronics \\ Research Institute, Tsukuba, Ibaraki 305-8568, Japan \\ ${ }^{4}$ National Institute of Advanced Industrial Science and Technology (AIST), department of \\ Electronics and manufacturing, Tsukuba, Ibaraki 305-8568, Japan \\ E-mail: roberto.cristiano@cnr.it
}

\begin{abstract}
We review progress in the development and applications of superconducting nano-strip particle detectors. Particle detectors based on superconducting nano-strips stem from the parent devices developed for single-photon detection (SSPD) and share with them ultrafast response time (sub-nanosecond) and operation at a relatively high temperature $(2-5$ $\mathrm{K})$ with respect to other cryogenic detectors. SSPDs have been used in the detection of electrons, neutral and charged ions, and biological macromolecules. Nevertheless, the development of superconducting nano-strip particle detectors has been mainly driven by the use in time-of-flight mass spectrometers (TOF-MS) where the goal of $100 \%$ efficiency at large mass values can be obtained. A special emphasis will be given to this case, reporting the great progress achieved which permits to overcome the limitations of existing mass spectrometers represented by low detection efficiency at large masses and charge/mass ambiguity and could represent a breakthrough in the field. In this review article we will introduce the device concept and detection principle, stressing the peculiarities of the nano-strip particle detector as well as its similarities with the photon detector. The development of the parallel strip configuration is introduced and extensively discussed, since it has contributed to significant progress in TOF-MS applications.
\end{abstract}

Keywords: Superconducting Detectors, Superconducting Nano-Strip, SSPD, Ions Detectors, Particle Detectors, TOF-MS 


\section{Contents}

1. Introduction

2. Superconducting Strip Particle Detectors (SSPDs)

2.1. Device concept

2.2. Particle detection with superconductors

2.3. Detection mechanisms: hotspot and vortex-based models

2.4. Tailoring the strip size for a specific application

\section{Meander-SSPDs}

3.1 TOF-MS with meander-SSPDs

3.2 Charge discrimination and true mass spectrometry

\section{Parallel-SSPDs}

4.1. Working regimes

4.1.1 The cascade or avalanche regime

4.1.2 The single-strip-switch regime

4.2 TOF-MS with parallel-SSPD

4.2.1 Comparison of Type-I and TypeII configurations

4.2.2 Type-II parallel-SSPDs with series resistors

4.2.3 Digitization of TOF values with superconducting TDC

\section{Conclusions}

\section{Introduction}

The history of superconducting particle detectors dates back to 1949 when Andrews et al.

[1] reported the detection of individual $\alpha$-particles using a superconducting strip. The phenomenon of superconductivity offers a variety of possibilities to realize radiation detectors, because the superconducting state is sensitive to various types of incident radiation in the form of both particles and electromagnetic waves. In the case of electromagnetic waves (photons) the covered electromagnetic spectrum is very wide and ranges from microwaves to $\gamma$-rays. Usually it is differentiated whether these detectors can discriminate single events, or if a constant flux of many photons sum up resulting in a measurement of the power. In this work we will confine our attention to the former type of detector which has the sensitivity and speed required to respond to a single quanta of 
incoming radiation, i.e. a single photon or a single particle. As quantum detectors for particles, superconducting devices are sensitive to $\alpha$-particles, electrons, neutrons, neutrinos or dark matter in a wide range of energy from $\mathrm{eV}$ to $\mathrm{MeV}$.

Superconducting detectors (SDs) are typically operated at temperatures close to absolute zero (the sub-Kelvin region) and thus have extremely high performance. Research in this field, which is still growing, has traditionally been driven by the need for high energy resolution in X-ray, visible astronomy and fundamental physics. Because the energy quanta associated with superconductors and lattice vibrations (phonons) are more than one hundred times smaller, substantial improvements have been obtained in energy resolution and in sensitivity over conventional detectors. Over the last three decades several types of SDs have been proposed and successfully used in experiments and scientific missions as well as for more practical applications. Among them we mention transition edge sensors (TES), kinetic inductance detectors (KID), superconducting tunnel junctions (STJ) and superconducting hot electron bolometers (S-HEB). Much of the original work in this field can be found in the proceedings of the Low Temperature Detectors Workshops that have been organized with a bi-annual periodicity since 1987 [2].

Thanks to the progress in the nanofabrication technology, in 2001 Gol'tsman et al. [3] demonstrated a new superconducting detector concept based on superconducting nanostrips. This type of device, known as the superconducting single-photon detector (SSPD) or superconducting nanowire single-photon detector (SNSPD), has a single-photon sensitivity in a range from visible to infrared wavelengths. SSPDs exhibit the remarkable property of recovery times and timing precision orders of magnitude faster than existing 
single-photon detectors based on semiconductors and superconductors. The ultimate response-time and sensitivity are obtained with ultra-thin thickness $(5-10 \mathrm{~nm})$ of the superconducting films, which are also patterned in very narrow nano-strips (so-called nanowires) of about $0.1-1.0 \mu \mathrm{m}$ width. In addition, SSPDs can operate at the boiling point of liquid helium $(4.2 \mathrm{~K})$ or a bit lower $(2.5-3.0 \mathrm{~K})$, a temperature range now within the reach of the rapidly improving cryogen-free cooler technology, which is cheap and user-friendly. SSPDs have been the subject of intense interest over the past decade and many research groups around the world are contributing to the development of this device. Significant advances have been made since their initial demonstration and SSPDs have now extremely fast response times with count rates beyond $200 \mathrm{MHz}$, timing jitter below $40 \mathrm{ps}$, low dark count rates in the order of $10 \mathrm{cps}$ and detector detection efficiency $>90 \%$ at a wavelength of $1550 \mathrm{~nm}$ by using an optical cavity [4-5].

In 2008 the use of an SSPD was proposed and experimentally demonstrated [6-7] in time-of-flight mass spectrometers (TOF-MS) as a detector of biological macromolecules with mass weight above $100 \mathrm{kDa}$ [8]. In TOF-MS a crucial requirement is the detector response time: sub-nanosecond rise time on the pulse leading edge, good timing jitter for high mass resolution and decay time below $10 \mathrm{~ns}$ for high counting rate. This is within the SSPD capability which has the further advantage of an almost $100 \%$ efficiency which is independent of the molecular weight and is expected to remain constant when the molecular mass increases. The use of SDs in TOF-MS dates back to 1996 [9-10]. The principal motivation at that time was the mass-independent efficiency provided by SDs which permitted to extend the sensitivity of modern MS instruments to very large mass 
values (above 1,000,000 Daltons), into the realm of the DNA fragments, proteins, and protein complexes. Unlike MS detectors based on secondary electron emission like microchannel-plates (MCPs) or secondary-electron-multipliers (SEMs), which are the off-theshelf technology for the TOF-MS, SDs do not rely on the secondary electron mechanism but are sensitive to phonons created by a single particle impact. A single impact of a heavy ion with a kinetic energy of tens of $\mathrm{keV}$ even with a low velocity may ultimately produce hundreds of thousands of phonons, which can be detected in a SD. In the past two types of SD were proposed and successfully used in TOF-MS applications: STJ [9] and hotelectron microcalorimeters [11]. Since mass spectrometry has become an increasingly important biomolecular research tool, this application represents a significant commercial target. Perhaps no tool has been as instrumental to the proteomics revolution as the mass spectrometer because these machines enable researchers to identify and quantify proteins. In fact, the basic mass spectrometer measures an ion's mass-to-charge $(\mathrm{m} / \mathrm{z})$ ratio. This enables peptide mass fingerprinting, which is the identification of a protein based on the specific group of peptide masses it produces. Motivated by the impressive impact of MS instruments in labs all over the world, efforts for the realization of TOF-MS instruments with superconducting detectors were immediately undertaken and a commercial version, the Micromizer, appeared in the market [12]. However, the progress in this direction became soon difficult, mainly because both STJ detectors and hot-electron microcalorimeters were characterized by a slow response time (microseconds) and sub-Kelvin temperatures $(100-300 \mathrm{mK})$ were necessary to operate the SDs. The cryogenic apparatus to reach such low temperatures was expensive and not user-friendly at that time. For this 
reason, TOF-MS instruments based on the SD-technology were not able to compete with conventional instruments.

SSPDs are an excellent candidate for TOF-MS because both the limitations in speed and operating temperature can be overcome. A serious drawback, however, was the very small size of a single-pixel because SSPDs rely on very narrow strips. In the very first proposal [7], the SSPD area was actually $50 \times 50 \mu \mathrm{m}^{2}$ only, smaller than STJs and far below the TOF-MS requirements. Significant progress has been made since that time and nowadays the SSPD detection area is more than three orders of magnitude larger, so that the typical molecular beam size $\left(\sim \mathrm{cm}^{2}\right)$ can be easily covered with an array of ten SSPDs [13].

The use of SSPD is not confined to TOF-MS applications, although this remains the main application of this device as a particle detector. In 2009 the Arndt Group in Vienna [14-15] presented a proof-of-principle study of SSPD for the detection of individual neutral molecules/nanoparticles at low energies. The detection of isolated neutral molecules and nanoparticles in the gas phase is a challenge for many experiments that range from physical chemistry and environmental monitoring, to the quantum matter wave interferometry of macroscopic objects [16]. While in TOF-MS one has to detect massive molecules (mass $>10^{5} \mathrm{Da}$ ) with a kinetic energy of $10-30 \mathrm{keV}$, similar to the energy of $\mathrm{X}$ ray photons, in this case the challenge is to detect relatively light neutral particles (mass < $\left.10^{3} \mathrm{Da}\right)$ with a small kinetic energy $(<1 \mathrm{eV})$, similar to the energy of mid-IR photons. In another study on the origin of the detection mechanism, SSPDs were also used to detect Argon ions $\left(\mathrm{Ar}^{+}, \mathrm{Ar}^{2+}\right.$, and $\left.\mathrm{Ar}^{3+}\right)$ accelerated with a voltage of $0.6-3.0 \mathrm{kV}$ [17]. Last, we 
mention the work of Rosticher et al. [18] that reports the detection of single-electrons with $\mathrm{keV}$ energies emitted from the cathode of a scanning electron microscope with an efficiency approaching unity. The variety of particles demonstrates the great versatility of SSPD as particle detector and motivates the interest in developing such device not only as a photon detector.

In spite of the fact that the device is practically the same, different acronyms have been used in literature when the SSPD was used as a particle detector: SSLD (superconducting strip-line detector) or SSID (Superconducting Strip Ion Detector) or SSED (Superconducting Single Electron Detector). The nomenclature of this type of devices is confusing, so that there is a demand for international standardization from the community. The problem has now come to the attention of the Technical Committee Working Group of the International Electrotechnical Commission (IEC TC90-WG14) which is working in collaboration with IEEE for naming superconducting sensors and detectors. According with their indication [19] the word "strip" or "nano-strip" may be suitable, since the ISO definition for "nanowire" applies only when the structure has similar cross-sectional dimensions $d$ and $w$ that differ in size by less than three times in a range of 1-100 $\mathrm{nm}$, which is not the case in our context. On this basis and taking advantage from the circumstance that the words "photons" and "particles" have the same initial, in this work we will adopt the acronym "SSPD" which in our case (particle detection) stands for Superconducting Strip Particle Detector. SNSPD (Superconducting Nano-Strip Particle Detector) will be also used to stress the use of a nano-structured superconducting strip. 


\section{Superconducting Strip Particle Detectors (SSPDs)}

\subsection{Device concept}

A SSPD is made up of many narrow strips (nanowires) of width $w$ and thickness $d$ placed side-by-side, and usually arranged in a meander geometry to increase the sensitive area and the collection efficiency, as shown in figure 1. The principle of operation of a superconducting single-particle detector essentially derives from its parent device, the superconducting single-photon detector and is based on a current-assisted mechanism. The nano-strips are typically cooled well below the superconducting critical temperature, $T_{\mathrm{c}}$, and biased at a current value slightly smaller than the critical current, $I_{\mathrm{c}}$. The energy of a single-particle hitting the strip surface generates a local zone of suppressed superconductivity. The subsequent energy cascade and production of excitations which leads to the detection response may be different in the case of photon or particle absorption. This aspect will be discussed later. For the moment, it is sufficient to say that a normally-conducting (non-superconducting) spot is produced at the location of the particle impact in a way similar to what happens in the case of photon absorption. The supercurrent is forced to flow around the normal zone, the so-called hotspot, through the two lateral sides which remained superconducting. Since the hotspot expands, the current density in the lateral sides of the strip increases and can reach the critical value. At this moment, a resistive region of the order of $\mathrm{k} \Omega$ appears over the entire strip cross-section and a large portion of the current is diverted out of the strip-line onto the electric load, $R_{\mathrm{L}}$, (typically $\sim 50 \Omega$ ) of the readout circuit, generating a voltage with a magnitude 
proportional to the diverted current. As the consequence of current deviation into the load, the current in the strip reduces and the Joule heating also decreases, then the hotspot collapses allowing the restoration of the superconducting state. The current can return back to the strip and the SSPD is ready for another detection event. The resistance and thus the response appears due to the collaborative effect of the bias current and the growing normal domain. The complete detection cycle is illustrated in figure 2. This detection mechanism requires narrow and thin nano-strips in order to guarantee that the entire cross-section of the strip becomes normal and the detection cycle takes place in a very short time. The nano-strip cross-section $(d \times w)$ can be adjusted in accordance with the amount of energy released by the impinging quantum of radiation: low energetic particles will require small cross-sections while larger cross-sections will be suitable for high energetic particles.

The SSPD is inserted in a bias and readout circuit as shown in figure 3(a). The DC bias current is typically supplied through a bias tee and the output pulses are readout by a room temperature amplifier via a coaxial load line which is schematically described here as a load resistance $R_{\mathrm{L}}$ placed in parallel to the SSPD. The external inductance $L$ takes into account the inductance of the wiring and the presence of a possible external inductance which can be mounted to slow down the fast output pulses. When a particle hits the strip and the normal region appears, the current is diverted into the load as shown in figure 3(b). The electrical equivalent of the SSPD is also shown in figure 3: an inductor $L_{\mathrm{k}}$, the kinetic inductance of the superconducting strip, in series with a parallel block made of a resistor $R_{\mathrm{n}}$, (the resistance of the normal domain) and a switch. When the strip is in the 
superconducting state the switch is closed and shunts the resistor (see figure 3(a)); after the arrival of a particle, the switch opens and adds the resistance $R_{\mathrm{n}}$ in series to the kinetic inductance $L_{\mathrm{k}}$ (see figure $3(\mathrm{~b})$ ). The voltage pulse across $R_{\mathrm{L}}$ has an amplitude equal to $I_{\mathrm{d}} / R_{\mathrm{L}}$, where $I_{\mathrm{d}}$ is the part of the bias current that was diverted from the SSPD onto the load. As first demonstrated by Kerman et al [20], the rise time and the decay time of the output voltage pulse are both proportional to the kinetic inductance of the strip:

$$
\begin{gathered}
\tau_{r}=\frac{L_{k}}{R_{n}+R_{L}} \\
\tau_{d}=\frac{L_{k}}{R_{L}}
\end{gathered}
$$

$L_{K}=\mu_{0} \lambda_{L}^{2} \frac{l}{w d}$, where $\lambda_{\mathrm{L}}$ is the London penetration depth of the superconducting material and $l$ is strip length.

\subsection{Particle detection with superconductors}

For a particle stopped on the surface of a superconductor, the predominant initial excitations produced by the energy transfer are phonons. In the case of SSPD as a detector of ions with keV energy as in TOF-MS, a possible scenario is described by Casaburi et al. [21]. On average the primeval phonons produced after the particle impact carry the Debye energy. The energy of these phonons is typically much smaller (tens of meV) than the energy needed to produce secondary electrons or electronic excitations $(\sim \mathrm{eV})$ as in conventional ionization detectors. Because of their short lifetime (1-2 ps), these phonons 
interact with the superconducting condensate via pair-breaking processes and create the normal region, the hotspot, with many highly excited electrons. In the subsequent tens of picoseconds ( $\sim 70 \mathrm{ps})$ the excited electrons diffuse, multiply, and decrease their excitation energy to about $3 \Delta$, where $\Delta$ is the superconducting energy gap [22-23]. At this stage the effective quasiparticle lifetime $\tau_{q p}$ is prolonged due to the phonon contribution and can be written as

$$
\tau_{q p}=\tau_{\mathrm{R}}\left[1+\left(\tau_{\mathrm{es}} / \tau_{\mathrm{ph}-\mathrm{e}}\right)\right]
$$

where $\tau_{\mathrm{R}}$ is the recombination time, $\tau_{\mathrm{ph}-\mathrm{e}}$ is the phonon-electron interaction time and $\tau_{\mathrm{es}}$ is the phonon escape time into the substrate [24]. The quasiparticle lifetime $\tau_{\mathrm{qp}}$ is thickness dependent because $\tau_{\mathrm{es}}=\kappa d$, where $\kappa$ is a constant that depends on the phonon transmission coefficient from the strip into the substrate [25]. Because the quasiparticle diffusion is much more extended than $d$, the perturbed volume is roughly cylindrical in shape with a radius $r=\left(D \tau_{q p}\right)^{1 / 2}$, where $D$ is the quasiparticle diffusion constant, and occupies an area $A_{\mathrm{qp}}=\pi r^{2}$ which scales linearly with $d$. Since the efficiency increases with $A_{\mathrm{qp}}$, the efficiency increases with the strip thickness $d$. This has been experimentally verified by Casaburi et al [21] and provides a support in favour of the described scenario. This result produces a marked difference with the situation when a SSPD is used for detection of IR photons where the efficiency decreases with the strip thickness.

In experiments with $\mathrm{He}$ ions performed at lower energy (see Table I) there was evidence that almost $99 \%$ of the incident ion energy is not transferred to the electronic system of the superconductor. [15] The authors pointed out the role of surface adsorbates that accumulate on the cold detector surface and act as a damping cushion. This problem 
of surface contamination can be common also to the case of TOF-MS, although is less relevant since no effects have been observed, probably because of the higher energy of the impacts and phonon propagation through the surface contaminant that is solid at low temperatures. In fact, it was reported that the phonons produced by the impact of $3 \mathrm{keV}$ acetyl-ions $\left(\mathrm{CH}_{3} \mathrm{CO}^{+}\right)$on the $700 \mathrm{~nm}$-thick $\mathrm{SiO}_{2}$ insulation layer that covered the top $\mathrm{Nb}$ electrode of an STJ broke Cooper pairs in the Nb electrode [26]. Moreover, the SSPD used in Ref. [15] had a thickness of only $4 \mathrm{~nm}$, and this, as shown above, may cause a decrease in the efficiency of the energy transfer process due to phonons leakage into the substrate.

\subsection{Detection mechanisms: hotspot and vortex-based models}

While progress has been made in understanding several aspects of SSPDs, many details of the detection mechanism in such detectors are still unknown. As illustrated in Section 2.2, it is thought that the result of the absorption of either a photon or a particle is the destruction of Cooper pairs and the production of a cloud of quasiparticles. [27-28] This process induced a breakdown of the superconductivity, resulting in a normal cross section. Currently, there is no consensus on a microscopic model based on the normal-core hotspot for the detection event in SSPDs as it was described in section 2.1. [29] In the case of photon detection, Renema et al. [30-31] gave a significant contribution to the discussion individuating different possible scenarios that could be responsible for the detection process. Following their argument, the first issue is whether the detection event occurs when the energy of the incident photon causes a cylindrical volume inside the strip to a transition to the normal state [3], or whether it is enough for the superconductivity to be 
weakened but not destroyed by the depletion of Cooper pairs over a more extended region.[27] The second issue is whether magnetic vortices play any role in the detection mechanism. There are two vortex-based models. The first is an extension of the normalcore model, where, a vortex-antivortex pair (VAP) forms at the point where the photon is absorbed [32]. In the second, the weakening of superconductivity lowers the energy barrier for either a vortex crossing [33-34] or a VAP crossing. In summary, the possible scenarios are four: 1) the normal-core hot spot model, where, the photon energy creates a normal domain inside the superconductor, which the current has to bypass; 2) the diffusion-based hot spot model, where, the quasiparticles diffuse outward from the point of absorption, creating a band of depleted superconductivity; 3) the vortex nucleation model, where, a VAP is formed in the hotspot; 4) the vortex crossing model, where, either a vortex or a VAP uses an area of weakened superconductivity to cross the strip and annihilate them. In the case of photons at optical frequencies the experiments of Renema et al [31] favours scenario 4) as the one that corresponds closest to reality and assigns to both quasiparticle diffusion and vortex unbinding a role in the detection event.

The above problem has been also investigated in the context of particle detection [17]. Experiments that measured the dependence of the detection efficiency on the bias current and the kinetic energies of Ar ions demonstrated that the hot-spot model governs the $\mathrm{keV}$ ion detection. This is understandable, as a single injection of a large amount of energy will be enough to break all the Cooper pairs at a single position along the strip, leading to a normal-core scenario. There must therefore be a typical energy where the diffusion-based scenario gives way to a normal-core scenario. The detection efficiency in the kinetic 
energy range of $0.5-9 \mathrm{keV}$ was well explained by the hot-spot model that assumes the proportional relationship between the hot-spot volume and the kinetic energies of Ar ions. Hence, it was suggested that the transition to the hotspot scenario should occur at an energy higher than a certain energy threshold which depends on the particular configuration and material used for the nano-strip [31]. The hotspot formation mechanism is valid for biomolecules that have more complex structures. More recently, in the case of molecular detection by $\mathrm{MgB}_{2}$ SSPD, time-dependent Ginzburg-Landau (TDGL) simulations coupled with a heat diffusion equation revealed that the hotspot model is relevant and the normal region expansion is completed within $16 \mathrm{ps}$, which corresponds to a maximum normal-region length of about $1000 \mathrm{~nm}$ for an energy deposition of $20 \mathrm{keV}$ [35]. The simulation was consistent with the experimental results of Lysozyme biomolecule ions.

\subsection{Tailoring the strip size for a specific application}

As we have seen in the introduction, SSPD can be used as a detector of very different particles: high energetic molecular ions in the case of TOF-MS, light and slowly moving neutrals molecules in the case of quantum interference experiments or high energy electrons. All types of particles considered so far, except electrons, are stopped at the surface of the superconducting film where they release their energy. This energy is converted in phonons which initiate the cascade to break Cooper pairs and produce the interaction. Problems related to reflection and inefficient absorption, which affect the photon detection, are therefore less relevant here. SSPDs present an absorption efficiency 
nearly equal to unity. Even with such an efficient absorption, the energy conversion factor can be less than unity since a consistent portion (of the order of $30-40 \%$ ) of the energy of the phonon system can be lost [11]. The important issue is how to retain a sufficient amount of energy in the form of phonons in the thin strip of a SSPD in order to produce a sufficiently large perturbation that can induce the detector response. In this sense, the nano-strip cross-section $(d \times w)$ must be tailored for the specific application. For instance, in the case of high energy particles the stringent constraints about the nano-sizing of the strip width and thickness can be relaxed and much thicker and wider strips can be used. Therefore, as described later, SSPDs with a recovery time of less than 1 ns and a large detector size over $1 \mathrm{~mm}$ can be realized with thicker and wider strips with the assistance of the parallel configuration. The strip cross-sections that were used for each specific application are listed in Table I.

\section{Meander-SSPDs}

\subsection{TOF-MS with meander-SSPDs}

TOF-MS is the main application of SSPD as particle detector. In MS, ionized analytes are accelerated by a static voltage $(\mathrm{V}=3-30 \mathrm{kV})$, acquiring a kinetic energy of $z e V$, where $e$ is the elementary charge and $z$ is the unit-charge number. The ions are separated either spatially or temporally, and are detected by an ion (particle) detector. The spatial or temporal separation by an electromagnetic force leads to the same result for any ion species having the same mass/charge $(\mathrm{m} / \mathrm{z})$ ratio. 
One type of the modern MS analyzers is based on time-of-flight (TOF) spectroscopy, which measures time lags between the acceleration and detection after field-free flight for a certain distance $\left(L_{\mathrm{TOF}}\right)$. Figure $4(\mathrm{a})$ shows a schematic of a TOF-MS with a MALDI (matrix-assisted laser desorption ionization) source where pulse laser shots ionize the analyte which was pre-treated, mixed with matrix molecules such as alpha-cyano-4hydroxycinnamic acid or synaptic acid, arrayed on a metal target plate, and allowed to dry. The plates enable high-throughput research, sometimes holding several hundred samples at once. Once an X-Y translation stage has positioned a specific spot, an ultraviolet laser strikes the sample. The matrix material absorbs this light energy, generating enough heat to vaporize and ionize peptide or protein molecules without dissociation, generally with a charge of $+1(z=1)$. The ions pass through extractor grids, ground plates and lenses to be focussed and then travels in a region of high electric field $(\mathrm{kV})$ where they are accelerated and launched for a free flight in a vacuum tube of length $L_{\mathrm{TOF}}$ which has on the opposite side the detector which measures each ion collision, producing a graph, or spectrum, of $m / z$ versus intensity. As an ion races down the flight tube, its speed is a function of its $m / z$ ratio. Hence, a particle's $m / z$ ratio is measured based upon its time of flight (TOF). The laser trigger or the high voltage pulse provides the starting time, while the detector signal gives the arrival time. The relationship between TOF and $\mathrm{m} / \mathrm{z}$ is expressed as

$$
T O F=L_{T O F} \sqrt{\frac{m}{z} \frac{1}{2 e V}}
$$

The first successful detection of biomolecules with an SSPD can be found in ref. [7] and [37] and the result is shown in figure 5, which shows the mass spectrum of a peptide, 
Angiotensin I, a standard sample for calibration with a mass weight of 1296 . The spectra were obtained by meandered-SSPDs with the sensor sizes of $200 \times 200 \mu \mathrm{m}^{2}$ and $50 \times 50$ $\mu \mathrm{m}^{2}$ that were mounted in a cryostat at the end of the TOF-MS flight tube. A practical instrument realized at AIST in Tsukuba, Japan, is shown in figure 4(b). The cryogen-free cryostat is added to a commercial instrument to cool down the SSPD. The picture shows the setup for the linear mode. Table II reports on the comparison between various meander-SSPDs with different areas and nano-strip cross-sections. It can be seen that the maximum area was of $200 \times 200 \mu \mathrm{m}^{2}$ and the best pulse rise time was 360 ps. These results were promising because of the good quantum efficiency, the fast response capability and the operating temperatures $(2-5 \mathrm{~K})$. Nevertheless, it was not easy to scale the detector area to larger sizes because the meander geometry is severely limited by the kinetic inductance that is proportional to the strip length: the largest SSPD had the worst response time. We will see in Section 4 how this limit can be overcome by a different SSPD architecture.

\subsection{Charge discrimination and true mass spectrometry.}

Some specifications for an ideal MS detector are listed in Koppenaal et al. [39] Among them there is no charge-state derivation because it is essentially impossible by using conventional MS instruments. All parts of conventional MS instruments have no ability to measure charge states $(z e)$. It is therefore impossible to distinguish ion species with the same $\mathrm{m} / \mathrm{z}$ value but different charge states ( $\mathrm{m} / \mathrm{z}$ overlap). In order to determine the $m$ values, speculation about $z$ values is required. Hence, a strict definition of mass spectra 
obtained with traditional MS instruments should be $\mathrm{m} / \mathrm{z}$ spectra, because the horizontal axis is $m / z$. It is worth to mention that the charge-discrimination problem can be solved by using STJ detectors [40], which can measure the kinetic energy of single molecules. The independent measurement of $E=z e V$ actually provides a tool to resolve the ambiguity of Eq.(2) and the mass can be obtained via the expression:

$$
m=\frac{2}{L_{T O F}^{2}} E \times T O F^{2}
$$

where the $T O F$ and $E$ values are obtained from two independent measurements.

Charge discrimination is an added value because it is possible to eliminate the charge/mass ambiguity of conventional instruments and realize a "true" mass spectrometer. SDs can solve this fundamental problem by measuring charge states: a typical example is seen in the ${ }^{14} \mathrm{~N}^{+}$and ${ }^{14} \mathrm{~N}_{2}{ }^{2+}$ ions, both of which have exactly the same $m / z$ value of 14,007 . The minority ions of ${ }^{14} \mathrm{~N}_{2}{ }^{2+}$ were never detected because the majority ions of ${ }^{14} \mathrm{~N}^{+}$masked the $m / z$ peak before the use of STJs. Detection of the doubly-charged ions is important for investigating atmosphere escape from planets. Thanks to the property of charge discrimination the interest for STJs is still alive [26], [40]. However, in an STJ the kinetic energy measurement is followed by the dead time of $1-20 \mu \mathrm{s}$, which is unreasonably slow for modern high-resolution time-of-flight analyzers. Suzuki at al. [41] demonstrated that, behind the nanosecond response, the short recovery time, the wide mass range, and low noise, SSPDs also possess the charge-state derivation ability. This was possible by subtracting data at different bias currents that determine detectable lower threshold energies of ions. The SSPD used in their work was a meander SSPD cooled to $4 \mathrm{~K}$. 
Molecule detection experiments were carried out by using chicken egg white lysozyme $(14,305 \mathrm{Da})$. The lysozyme molecules were ionized by a MALDI source that produced mainly singly- and doubly-charged ions, and a negligible amount of triply-charged ions. The ions were accelerated by a static high voltage of $17.5 \mathrm{kV}$, and acquired a kinetic energy of $17.5 \times z \mathrm{keV}$. In this study, they found that the charge-state derivation with the SSPD was possible by simply changing the current bias points, as shown in figure 6 . As briefly mentioned in section 2.3 , the charge state derivation by SSPDs can be understood by the hotspot model, in which the diameter of hotspots are proportional to the square root of the kinetic energies of ions. [17] The curves of the detection efficiency as a function of bias current show abrupt reductions at certain bias currents that depend on the ionic charge states and are defined as threshold bias currents $\left(I_{t h}\right)$ for detecting ions. The experimental $I_{\text {th }}$ was found to follow $I_{t h} / I_{c}=1-(z e V)^{1 / 2} \mathrm{C} / \mathrm{w}$, where $C$ is the factor determined by the density of states at the Fermi level in principle. Therefore, for example, it is possible to detect ions with $z$ values more than 2 by adjusting the bias point. By subtracting two spectra measured at different bias points, it is possible to obtain the ion intensity for each charge state.

\section{Parallel-SSPDs}

As is often the case in applied nanotechnology, devices based on nano-structured elements have to reach micro-meter (SSPD for photon detection applications) or even millimetre (SSPD for particle detection in TOF-MS applications) size to be comparable with either 
the core diameter of optical fibers or the diameter of molecular beams in TOF-MS. For this reason, the nano-strips are assembled with a meander shape, as the one shown in figure 1. Since the SSPD is attractive for its fast response time, the challenge was to lengthen the nano-strips to achieve areas as large as possible without degradation of the sub-nanosecond response time. The meander architecture has an intrinsic limitation because the kinetic inductance $L_{\mathrm{k}}$ linearly increases with the strip length and, as shown by Eqs. (1a) and (1b), this leads to an increase of both the rise-time $\tau_{\mathrm{r}}$ and the decay time $\tau_{\mathrm{d}}$ of the output pulse signals. The problem is to find an alternative SSPD architecture that retains the fast response when the length of the nano-strips increases.

A smart solution to overcome this inconvenience is provided by the pure parallel architecture (we call it the Type-0 configuration) as proposed by Ejrnaes et al. [42-43] where two or more nano-strips are assembled in parallel. In figure 7 the schematics of the various SSPD architectures are reported: the basic building block constituted by the elementary single-strip (figure 7(a)), the meander configuration (figure 7(b)) and three types of parallel configurations. The pure parallel architecture (Type- 0 configuration) is shown in figure $7(\mathrm{c})$. In addition to the original Type- 0 configuration, in literature two additional types of parallel-nano-strips architectures have been developed: Type-I and Type-II. They are schematically shown in figures $7(\mathrm{~d})$ and $7(\mathrm{e})$ and their practical realizations are shown in figures $7(\mathrm{f})$ and $7(\mathrm{~g})$. For a meaningful comparison between the various configurations, including the meander, it becomes useful to introduce $L_{0}$, the kinetic inductance of the elementary single-strip (figure 7(a)), which can be considered as the basic element of any SSPD configuration. Assuming a length $l_{0}$ for the elementary 
single-strip, its kinetic inductance is $L_{0} \approx \mu_{0} \lambda_{L}^{2} \frac{l_{0}}{w d}$. Since a meander (figure 7(b)) can be considered as a connection of $n$ elementary single-strips in series, its kinetic inductance is:

$$
L_{k}^{\text {meander }}=n \times L_{0}
$$

In a Type- 0 configuration as shown in figure $7(\mathrm{c})$, the same number of elementary singlestrips of a meander are assembled in parallel so that the kinetic inductance obviously reduces to $L_{k}^{\text {parallel }}=\frac{L_{0}}{n}$ or $L_{k}^{\text {parallel }}=\frac{1}{n^{2}} L_{k}^{\text {meander }}$. According with Eqs. 1(a) and 1(b), this means that the same number of strips will cover the same area but the parallel configuration will produce a device which is $n^{2}$ times faster than the meander. The improvement in speed is not the only favourable property of the parallel configuration, as it will be clear in next sections.

In Type-I configuration of parallel strips [43], $n_{\mathrm{P}}(>1)$ parallel nano-strips form a single-block and the SSPD area is compiled from $n_{\mathrm{B}}(>1)$ blocks in-series-connected. This configuration mimics the serial connection used in the meander-SSPD, with the difference that each element has $n_{\mathrm{P}}$ times lower inductance while it covers $n_{\mathrm{P}}$ times more area. In the limiting case of $n_{\mathrm{B}}=1$, one has the "pure" Type- 0 configuration, whereas if $n_{\mathrm{P}}$ $=1$ one has the "pure" meander configuration. In Type-II SSPD [44], the same area is compiled by folding $n_{\mathrm{S}}$ nano-strips in meander fashion and $n_{\mathrm{P}}$ meanders are then connected in parallel to realize the same active area. Both the architectures should have the same performances in terms of response time and signal amplitude, given that the total 
number $n$ of single-strips is the same, each elementary strip has the same length $l_{0}$ and is characterized by the same critical current $I_{\mathrm{C}, 0}$. In this case, the following relationships

$$
\begin{aligned}
L_{k}^{\text {parallel }} & =\frac{1}{n_{P}^{2}} L_{k}^{\text {meander }} \\
V_{\text {out }} & \propto n_{P} I_{C, 0}
\end{aligned}
$$

hold independently from the particular architecture. The overall behaviour of these two types of architectures can be different, however, for what concerns the possible working regimes and the aspects related with the latching phenomenon, as it will be described in the next sections.

\subsection{Working regimes}

A parallel SSPD can work in different regimes. The most popular is the cascade or avalanche regime [42-44] which provides not only high speed but also signal amplification. The cascade is triggered when the bias current is above a certain threshold value and stems from the redirection of the bias current from the struck nano-strip into the other parallel nano-strips. At lower current values, a click from the detector can also be recorded, due to the collaborative effect of an event absorbed in a nano-strip, which arms the remaining parallel strips, which then clicks at a second event absorption. Again this latter can trigger a cascade so that the arm-and-trigger regime can be viewed as a subcategory of the cascade regime.

Very different from the cascade is the single-strip-switch regime where avalanche does not occur and the click is produced by a portion of the bias current from a single strip 
switched to the normal state by an event absorption. [45-46] This regime was observed in parallel-SSPDs with large nano-strips cross-sections that carry such a high current which was sufficient to produce a voltage signal across the load resistance with an amplitude well above the noise level. Cascade and single-strip-switch regimes will be described in Sections 4.1.1 and 4.1.2.

\subsubsection{The cascade or avalanche regime}

In the cascade switch mechanism [42], the electronic signal pulse induced by photon/particle absorption in a single nano-strip is generated when the switch to the normal state occurs in cascade in all the parallel nano-strips. The critical issue is to find the proper configuration that guarantees the occurrence of the cascade switch. For the sake of simplicity, let us refer to the Type-0 architecture of figure 7(c). In detail, all nano-strips are biased with a current slightly below their critical current. When one nano-strip switches to the normal state after absorbing a particle (initiating nano-strip), part of its current is redistributed among the still-superconducting nano-strips in parallel (secondary nano-strips) and $R_{\text {load. }}$ The cascade occurs if the redirect current is sufficient to overcome the critical current of each individual nano-strip so that an avalanche is produced and the entire bias current is diverted into the load. To properly trigger the cascade switch of all the secondary nano-strips, at the initial stage that follows the switch of the initiating nanostrip it is necessary that the current remains confined in the parallel nano-strips and is not immediately diverted into the load. This condition can be obtained by inserting an inductance $L_{\mathrm{s}}$ in series with the block of parallel nano-strips to confine the current during 
the initial current redistribution. Since the inductance has the role of preventing that current is diverted into the load, it is sufficient that its value is roughly equal to the inductance of a single strip $L_{0}$. Moreover, if the bias current is higher than a certain minimum threshold current, $I_{\mathrm{thr}}$, the redistributed currents will induce the switching to the normal state in all the parallel nano-strips. At this point, the bias current will finally deviate onto the load and the signal appears. Since the deviated current is the sum of the currents in all the parallel nano-strips, a large signal is produced. When the current is finally flowing through the load rather than in the detector, the Joule heating in the nanostrips decreases. This allows the cooling to restore the superconducting state, which will make the current return into the SSPD. The balance between the cooling power and the speed of current return to the SSPD is responsible for restoring the sensitivity again. The optimal balancing can be realized again by a proper choice of detector inductance.

The presence of $L_{\mathrm{s}}$ is important for reliable triggering of the cascade switch mechanism. If too low an inductance value is used the probability that a particle impact event does not trigger the cascade switch will increase. When the cascade switch does not occur the parallel strips are no longer homogeneously biased. The change of the bias current distribution among the parallel strips will be discussed in section 4.2.2. This negatively affects the subsequent performance of the detector because the strips with different bias levels will respond with different efficiencies.

In the cascade regime, $L_{\mathrm{s}}$ will also increase the overall detector inductance, with a negative effect on the maximum signal repetition rate. When there is a need for the cascade regime and an extra inductance is necessary, the latter can be realized with other 
blocks of parallel nano-strips (Type-I architecture of figure $7(d)$ ). In this way one also simultaneously contribute to an increase of the total area of the parallel-SSPD since the inductance is made of active detection elements. To obtain the optimal performance, a proper choice of the number $n_{P}$ of parallel nano-strips in a block and the number $n_{B}$ of blocks must be made [47].

As described above, in the cascade or avalanche mode the signal amplitude is the sum of the currents in all the parallel nano-strips. Hence, one obtain assort of signal amplification, which is proportional to the number of parallel nano-strips $\left(V_{\text {out }} \propto n_{P} I_{C, 0}\right)$. This intrinsic amplification is very attractive in applications when there is a need to push the SSPD sensitivity into the sub-eV region of longer wavelengths to detect far infrared photons or to detect very low energetic particles. In this cases, better sensitivity can be obtained by decreasing the nano-strip's width: SSPDs based on few-tens-of-nanometerwide strips are expected to efficiently detect mid-infrared photons at longer wavelengths $(\sim 3000 \mathrm{~nm})$ for instance. However, the reduced strip cross-section will result in lower bias currents and in turns in a degradation of the signal-to-noise ratio because the output signal amplitude is proportional to bias current value. The parallel architecture working in the cascade regime provides better signal-to-noise ratio and overcomes this limitation as suggested by Marsili et al [44].

An extensive investigation of the cascade switch (or avalanche) regime was made in a series of papers by Marsili et al. [44], [48], [49]. Although these works refer to the Type-II architecture, most of their results apply as well to the other case. Ideally, no current from the initiating strip should be diverted to the load before the secondary parallel nano-strips 
have switched to the normal state (this ideal operation mechanism is called perfect redistribution). However, in practice, the current leaking, $I_{\text {leak, }}$, to $R_{\text {load }}$ can be substantial. It was found that, depending on $r$ (the ratio between $I_{\text {leak }}$ and the current redistributing to all the secondary nano-strips $\left.r=\frac{L_{0}}{L_{s}\left(n_{P}-1\right)}\right)$ and on $I_{\text {bias}}$, devices could exhibit: (1) correct operation as single-particle detector (avalanche regime); (2) operation in arm-trigger regime as two- or $n$-particle detector. In ref. [44] and [48] it was suggested that for $I_{\text {bias }}>$ $I_{\mathrm{thr}}$, each hot-spot nucleation event triggers an avalanche. For $I_{1}<I_{\text {bias }}<\mathrm{I}_{\mathrm{thr}}$, we are in the arm-trigger regime where two subsequent hot-spot nucleation events are necessary to create one avalanche. According to the analysis of the device operation conducted in ref [42] perfect redistribution implies that the cascaded switch works in the current bias range $I_{\mathrm{C}}\left(n_{p}-1\right) / n_{p}<I_{\text {bias }}<I_{\mathrm{C}}$. To take into account the fact that in practice it may happen that $I_{\text {leak }}$ $\neq 0$, a determination of $I_{\mathrm{thr}}$, can be obtained from the electro-thermal model [44], [48].

The series inductance $L_{\mathrm{s}}$ influences the electrical time scale of the SSPD. This is true independently if it was realized as an added external inductance or by means of the Type-I architecture. Indeed, when the electrical and thermal-relaxation time scales become comparable, superconducting-nano-strip-based detectors appear to malfunction by either latching or afterpulsing, depending on the detector architecture and electrical environment [49-50]. Latching occurs when the SSPD does not return to the superconducting state after a detection event. The occurrence of latching effectively stops the detector from responding to subsequent particles arrivals, even if the bias current is lower than the critical current. Latching is detrimental because it inhibits detector operation in the bias 
region of high efficiency. This phenomenon is also observed in meander-SSPD, but parallel-SSPDs are particularly susceptible to latching, probably due to the fact that operation is faster while energy dissipation in the detector is higher. Experimentally, this manifests itself as a need of large detector inductance values as increased $n_{\mathrm{P}}$ is used, to achieve proper operation [43]. By realizing the inductance with sensitive nano-strips (Type-I) one can achieve fast operation of large area detectors. Latching in parallel-SSPDs can be avoided, for a fixed load of $50 \Omega$, by properly choosing the number $n_{\mathrm{P}}$ of parallel nano-strips according to the needed detector area. Smaller detectors use smaller $n_{\mathrm{P}}$ whereas larger detectors can use more parallel nano-strips without triggering the latching phenomenon. It is also likely that larger $n_{\mathrm{P}}$ can be used if the load resistance is reduced. According to ref. [51], this could make the SSPD faster because the inductance can be reduced more than the load thereby lowering the time constant that limits the return of the current into the detector. This possibility comes at the cost of reduced signal pulse amplitude and therefore also reduces the signal-to-noise ratio of the detector signal. Afterpulsing in parallel-SSPDs is likely due to the thermal relaxation dynamics of the superconducting nano-strips. This phenomenon was investigated by Marsili et al. [49], and is the consequence of the more or less fast recovery of the superconducting state in the nano-strips. The effect of reducing the $L_{\mathrm{s}}$ value to make the SSPD fast was investigated, and it was observed that parallel-SSPDs with low $L_{\mathrm{s}}(r>0.1)$ emitted trains of current pulses when biased at a lower current than the onset of the arm-trigger regime. Moreover, the desired decrease in the detector reset time came at the price of an increase in the avalanche current, which decreased the bias range for the correct operation of the devices. 
Below a certain limit shorter reset times produce afterpulsing. This can be due to the device materials and geometries: therefore, engineering the thermal environment of the superconducting nano-strips (by properly choosing the substrate material or surface preparation or by patterning thermally conductive materials on the nano-strips) may result in a decrease of the thermal-relaxation time, which is required to allow reducing the detector reset time. Worthy of note is that afterpulsing was never observed in TOF-MS experiments with a parallel-SSPD.

\subsubsection{The single-strip-switch regime}

Parallel-SSPDs with Type-I architecture has been extensively used in the development of large area detectors for TOF-MS. In this investigation it turned out that device could not be operated in the avalanche mode because after any detection event the device latched. Anyway, as the diverted current from the hit strip onto the load is sufficiently large it was not necessary operates it in the avalanche mode and the detector could have been conveniently operated in the so-called single-strip-switch regime. To understand the reason for this choice one has to note that the achievement of large areas with parallelSSPDs was accomplished both by increasing the total number of strips and the strip crosssection $(w \times d)$. As the consequence, the absolute value of the bias current flowing thorough a single strip as well as the absolute value of the total bias current flowing in the device were very large. For instance, in ref. [46] the SSPD was formed by 101 series connected blocks, each of which contains 5 parallel striplines with a cross-section of $1 \mu \mathrm{m} \times 40 \mathrm{~nm}$ 
(see figure 8). This device had a critical current (maximum switching current to the resistive state) $I_{\mathrm{c}}$ value of $17.1 \mathrm{~mA}$ which corresponds to $I_{\mathrm{C}, 0} \sim 3.4 \mathrm{~mA}$. A latching current ( $I_{\text {latch }}$ of $9.3 \mathrm{~mA}$ (as low as $54 \%$ of $I_{\mathrm{c}}$ ) was observed. To operate the SSPD a low bias current of $9.0 \mathrm{~mA}\left(97 \%\right.$ of $\left.I_{\text {latch }}\right)$ was sufficient and this guarantees the spontaneous return to the superconducting state. The analysis of the observed lower pulse amplitudes showed that the output pulses were produced by the superconducting-normal transition within only one of the parallel nano-strips instead of cascade switching of all the parallel nano-strips. This is reasonable because the current diverted into the load impedance of the read-out was only $0.01-1 \%$ of the current flowing in a single nano-strip that a particle impinged upon, but it was sufficiently large to overcome the noise level. Part of the current is also diverted into the neighbouring parallel strips of the block but it was not sufficient to trigger the cascade switch. On the other hand, the operation at higher bias current to enable the cascade regime is useless because it would lead to latching. As we have seen, in the single-strip-switch regime (also referred as partial switching) only the impacted strip is driven into the normal resistive state while the other strips in the detector remain in the superconducting state. This occurs when $I_{\text {bias }}$ is smaller than the threshold value for cascade triggering.

In the single-strip-switch regime the generated output pulses have non constant amplitudes varying over a large range of values, a circumstance that indicates a nonuniform current distribution among the parallel strips. The bias current in the impacted nano-strip probably does not recover its initial value when superconductivity is restored after a detection event. The diverted current is redistributed as an extra current among the 
$n_{\mathrm{P}}-1$ parallel nano-strips of the block. After some detection events, a non-uniform distribution of the bias current is established among the strips in the block with the consequence that some strips are biased at lower and others at higher currents compared to the initial bias current distribution. In recent papers [52-53], both the current recovery after detection events and the current redistribution among parallel strips after output pulse triggering have been investigated in order to gain insight into the factors governing the output pulse amplitude and explain the considerable spread in the pulse amplitude distribution [13], [46]. The effect of the current redistribution among parallel strips after each detection event could also explain why the efficiency of the detectors it does not increase at increasing of the sensitive area as pointed out in [52]. The experiments used a nano-optical technique, which probed individual nano-strips in an especially designed SSPD with intense laser shots. The ad-hoc geometry consisted of six parallel strips spaced such that they could be individually illuminated. The measurements demonstrated that the initial current distribution was not uniform but governed by the London theory, with a typical penetration profile dominated by the magnetic penetration depth. Moreover, the key characteristic of the single-strip-switch regime was that the fired nano-strips recovered the superconducting state after firing but did not recover the initial bias current. Therefore, the nano-strip generated only one pulse and then became insensitive to further particle arrivals unless the bias current was reset or several other nano-strips were fired. From energetic consideration of the current and the auto-induced magnetic field in the device and by observing the distribution of generated pulse amplitudes, there is evidence that the London model governs the current redistribution in the parallel strips also after the initial 
nano-strip switch event. These studies serve as a useful guide for the future development of the next generation of high efficiency parallel SSPDs with large active areas for TOFMS applications. It is worth mentioning here that the possible integration of resistors in series with the parallel nano-strip may represent a viable solution to restore uniformity and increase the bias current level to obtain higher efficiency. Experimental results of SSPDs with series resistors will be described in the section 4.2.2.

A comparison between Type-I and Type-II architectures is mandatory to investigate the subtle differences between these two architectures that apparently should have a similar behaviour. A first attempt in this direction was made by Zen et al [54]. They reported experiments that compared devices with Type-I and Type-II architectures, in the case of ion detection. Two Nb-based devices were fabricated in the same conditions but patterned in different configurations. In spite of the fact that the two architectures should exhibit the same response time and critical currents, several differences were observed. The Type I device showed a faster response time and a lower critical current which means pulses of smaller amplitudes. Moreover, analysis of the distribution of the pulse amplitudes showed that Type I architecture was affected by a considerable loss in ion counting. These phenomena will be described in the section 4.2.1. This phenomenology highlights the complexity of the parallel architectures in terms of the dynamical behaviour of the current which is diverted from the SSPD into the load resistor and the resulting redistribution of the bias currents flowing through the parallel nano-strips.

Finally, the current non-uniform redistribution can result in a different efficiency for lowly- or highly-energetic ions. In fact, nano-strips with low bias current will become 
sensitive to double charged ions $(z=2)$ while nano-strips with a higher bias energy will likely respond to the arrival of singly charged ions $(z=1)[41]$. It has been hence envisaged that the phenomenon of efficiency change could enable charge discrimination in parallel-SSPDs [13].

\subsection{TOF-MS with parallel-SSPD}

The TOF-MS application is the most demanding for response time and area coverage. Widely used ion detectors such as MCPs or SEMs have a nanosecond ion-counting capability necessary for a resolving power $(\mathrm{m} / \Delta \mathrm{m})$ over 10,000 , and a $\mathrm{cm}$ size covering the molecular beam. As described in Section 3, the first SSPD for TOF-MS had a sensitive area of $50 \times 50 \mu \mathrm{m}^{2}$ and showed a $\tau_{\mathrm{r}}$ of 360 ps and pulse decay time, $\tau_{\mathrm{d}}$, of $9 \mathrm{~ns}$ [7]. However, with such a small device area, an array of about $4 \times 10^{4}$ SSPD pixels is necessary to cover the desired area of $1 \mathrm{~cm}^{2}$ of the molecular beam and this large number of pixels is hard to handle in a compact cryogenic apparatus. Attempts to increase the SSPD area to $200 \times 200 \mu \mathrm{m}^{2}$ [38] by using a larger number of longer strip-lines caused $\tau_{\mathrm{r}}$ to increase up to $1 \mathrm{~ns}$. In 2009 Casaburi et al. [45] first proposed the use of parallel-SSPD with a Type I architecture as a proof of principle device to demonstrate the capability of this configuration. In this work an area of $200 \times 200 \mu \mathrm{m}^{2}$ was covered by assembling $n_{\mathrm{B}}=$ 39 blocks in series, each having five $\mathrm{Nb}$ parallel nano-strips $\left(n_{\mathrm{p}}=5\right)$. The measured response times of the signal pulses were $\tau_{\mathrm{r}}=400 \mathrm{ps}$ and $\tau_{\mathrm{d}}=500 \mathrm{ps}$, much faster than a similar meander fabricated in the same condition which had $\tau_{\mathrm{r}}=10 \mathrm{~ns}$ and $\tau_{\mathrm{d}}=26 \mathrm{~ns}$. Soon after, in ref. [46] it was demontrated the good performance of a parallel-SSPD with 
the remarkable area of $1 \times 1 \mathrm{~mm}^{2}$. The device consisted of 51 series connected blocks, each with 10 parallel strips. The response times were $\tau_{\mathrm{r}}=520 \mathrm{ps}$ and $\tau_{\mathrm{d}}=920 \mathrm{ps}$, still in the sub-nanosecond region. A spectrum of the multimers of immunoglobulin G (IgG, 146 $\mathrm{kDa}$ ) obtained with this device is shown in figure 9. It confirms the detection capability in a molecular weight range from 1000 to 600000 . The inset helps in the visualization of the peaks at large mass values by plotting a portion of the spectrum in a logarithmic scale. The peak intensity decreases with the TOF, reflecting the fact that the abundance of the multimers in the molecular beam decreases as the multimerization number increases. The recognizable IgG multimer peaks up to tetramer $(584 \mathrm{kDa})$ indicating high sensitivity for large molecules. The largest area obtained so far with parallel-SSPDs is $2 \times 2 \mathrm{~mm}^{2}$ [13], [54] while keeping $\tau_{\mathrm{r}}=500 \mathrm{ps}$ and $\tau_{\mathrm{d}}=9 \mathrm{~ns}$ [13]. A TOF-MS spectrum obtained with such

a device with Type-I architecture is shown in Figure 10. The tested molecule was lysozime $(14.3 \mathrm{kDa})$. The spectrum was obtained in 20 minutes with 2400 laser shots. The peaks are labelled with the symbols $\mathrm{M}^{+}$and so on that indicate the multimer base of the molecular complex and the corresponding charge state. The spectrum shows peaks up to $10 \mathrm{M}^{+}$which corresponds to mass weight of $143 \mathrm{kDa}$.

\subsubsection{Comparisons of Type-I and Type-II parallel configurations}

Despite the same cross section of strips and the same number of parallel strips, detection properties of Type-I and Type-II configurations are different in several points [54]. First, $I_{c}$ of Type-I is about half of that of Type-II. Second, the time constant in Type-I is faster than that in Type-II. Although the origins of these phenomena are still under investigation, 
the different geometric structure should play an important role in these differences. For example, a self-magnetic field created by the bias current in a superconducting strip is cancelled in Type-II (see figure 7(e)) where the strip has the going and returning configuration, while it is not cancelled in Type-I (see figure 7(d)). In addition, the output pulse height of Type-I is 1-2 orders of magnitude smaller than that of Type-II. Because a single block of Type-I consists of short strips in parallel (figure 7(d)), its kinetic inductance is so small that the output current easily diverts inside of the block. Therefore, a small portion of the output current flows to the load resistor and the output pulse height becomes small. Some of them are so small that they are buried under a noise level, which results in the ion count loss. On the other hand, an output pulse height of Type-II is large enough above the noise level. Because Type-II consists of the long meander strips arranged in parallel, of which the kinetic inductance is large (figure 7(e)), a sufficient amount of output current flows to the load resistor. It is noteworthy that no preamplifiers are necessary for Type-II SSPDs.

The pulse heights of output signals of Type-II widely distribute, as is expected from the initial bias current distribution determined by the London theory [52-53]. Circuit simulations reveal that the wide distribution of output pulse heights is caused by the redistribution of the bias current among parallel strips after each ion impact, as mentioned before. For a larger bias current, the redistributed current switches some of neighboring parallel strips that encounter no ion impact. Further increasing of the bias current results in the cascade switching of all parallel strips, resulting in the latching in the normal state and no sensitivity for particle impacts. Thus, the cascade switching is undesirable for TOF-MS. 
Consequently, it is difficult to realize $100 \%$ detection efficiency for parallel SSPDs as long as the cascade switching occurs in high bias currents.

\subsubsection{Type-II parallel SSPDs with series resistors}

The circuit simulation has proven that a resistor serially connected to each parallel strip is effective to prevent the redistribution of the bias current, as shown in figure 11 [54]. Using resistors, the redirected bias current returns to the same strip in a certain time. The recovery time is expressed as:

$$
\tau_{\text {recover }}=L_{0} / R_{0}
$$

where, $R_{0}$ is the series resistance. The simulation was conducted with and without small bias resistors $R_{0}=1 \Omega$ connected in series to all strips, as shown in figure 11(a). The bias resistors are expected to suppress the current diverted into the neighbouring parallel strips $I_{\text {loss. }}$ Figure $11(\mathrm{~b})$ shows the time evolution of the current in a single strip $I_{\mathrm{SW}}$ with and without $R_{0}$. In both cases, initial $I_{\mathrm{SW}}=I_{\text {bias }} / 10=3 \mathrm{~mA}$, assuming that $I_{\text {bias }}=30 \mathrm{~mA}$. Without $R_{0}$ (red lines), the $I_{\mathrm{SW}}$ losses are not recovered until the other parallel strips switch. However, with $R_{0}, I_{\mathrm{SW}}$ rapidly recovers to $3 \mathrm{~mA}$ immediately after each ion event (blue line). The histograms of $I_{\text {load, }}$, the current diverted into the load, are compared in figure 11(c), where 1973 and 1498 ion events are simulated in the presence (blue) and absence (red) of $R_{0}$, respectively (normalized by peak count). Without $R_{0}$ (red), the histogram is broad. Conversely, with $R_{0}$, the histogram is sharp, indicating that $I_{\text {loss }}$ is effectively suppressed, as is the case with the SSPD in a single meander configuration. 
For the first trial, the resistors are realized in the aluminum bonding wires. The pulse height distribution exhibits an improvement, which is attributable to the suppression of the bias current redistribution induced by particle impact [55]. However, $R_{0}$ of the bonding wire was so small that $\tau_{\text {recover }}$ was long, so the bias current couldn't recover its initial value before subsequent ion impacts. Therefore, in high-count-rate measurements, the pulse height distribution became broad. To avoid the bias current dynamical redistribution even in high-count-rate measurements, a faster $\tau_{\text {recover }}$ is mandatory; that is, a larger $R_{0}$ is necessary. However, a simple connection of the large resistances to superconducting strips needs to be avoided because the bias current of the SSPDs is in the range of $\mathrm{mA}$ and the corresponding Joule heating results in a temperature increase of SSPDs. To overcome this difficulty, new biasing schemes are under development.

\subsubsection{Digitization of TOF value with superconducting TDC}

Although the current parallel SSPDs have a fast response time ( ns) and cover a large area $\left(2 \times 2 \mathrm{~mm}^{2}\right)$, the size is still small compared to the diameter of an ion beam of $\sim 1 \mathrm{~cm}$. One solution is setting a parallel-SSPD in an array, but multiple readout lines for $\mathrm{GHz}$ bandwidths induce a fatal thermal inflow that leads to a rise in the base temperature. In order to overcome this problem, Sano et al. [56] are developing the use of time-to-digital converter (TDC) based on the single flux quantum (SFQ) technology.

The SFQ TDC can produce digital data for TOF values of ions for all SSPD pixels at the low temperature stage, and therefore only one slow readout line between the $4 \mathrm{~K}$ stage and room temperature is necessary to transfer the TOF digital data to room temperature 
electronics. The SFQ-TDC contains several thousands of Josephson junctions for digitization and buffering. Currently, the SSPD chip and the SFQ-TDC chip are separated. In future, both chips could be integrated in a single chip in order to realize the detector size of $\sim 1 \mathrm{~cm}$.

\section{Conclusions}

Superconducting nano-strip particle detectors represent an interesting device for several applications because they are able to detect charged and neutral ions, massive and lightweight particles in a wide range of kinetic energies. The variety of particles demonstrates the great versatility of the SSPD as particle detector. In this work we have discussed the working principle, stressing the peculiarity of particles' interactions with superconducting nano-strips and the consequence that this may have on the design of practical devices. The case of TOF-MS application has been discussed in great detail because nowadays it is the driving application. Great progress has been made in this direction which evidences the SSPD as a mature technology. SSPDs for OF-MS have subnanosecond response time, single-pixel area of $\mathrm{mm}^{2}$, practically $100 \%$ efficiency up to MDa mass values and operate at $2-5 \mathrm{~K}$. The high working temperature of the SSPD is certainly an attractive property of this device. $\mathrm{Nb}$ and $\mathrm{NbN}$ have been so far the materials of choice. The recent progress with high critical temperature $\mathrm{MgB}_{2}$-based SSPDs opens new and more stimulating perspectives to push the working temperature above $10 \mathrm{~K}$ [35]. This, together with the high efficiency already demonstrated by the SSPD at extremely 
large masses and the possibility of direct charge discrimination represents a milestone in TOF-MS that could revolutionize the field. Moreover, the possibility of detecting neutral and labile molecules is also promising for many applications in physical chemistry and matter-wave interferometry. Superconducting nano-strip particle detector technology certainly requires further development and exploration but it represents a positive opportunity for detection and investigation of particles which are too complex to be well detected by other means.

\section{Acknowledgments}

The authors would like to express thanks to S. Shiki, G. Fujii, K. Suzuki, K. Sano, N. Yoshikawa and M. Ukibe for their assistance and fruitful discussion. A.C. thanks Marie Curie Actions, the staff of the James Watt Nanofabrication Centre at the University of Glasgow and support from the UK Engineering \& Physical Sciences Research Council. N. Z. acknowledges a Grant-in-Aid for Scientific Research (C) from the Japan Society for the Promotion of Science (Grant No. 24619013). The authors thank Barbara Pizzicato for figure 4(a).

\section{References}

1. Andrews D H, Fowler R D, Williams M C, 1949, Phys. Rev. 76, 154

2. The series of Low Temperature Detectors LTD-proceedings (from LTD-1 to LTD-15) provides a good source of information about superconducting detectors. For a modern overview see also the volume Applied Superconductivity: Handbook on Devices and Applications, Paul Seidel (Editor), Wiley-VCH publisher, February 2015, Chapt. 8 Superconducting Radiation and Particle Detectors, ISBN: 978-3-527-41209-9

3. Gol'tsman G N, Okunev O, Chulkova G, Lipatov A, Semenov A, Smirnov K, Voronov B, Dzardanov A, Williams C and Sobolewski R, 2001 Appl Phys. Lett., 79, 705 
4. see the topical review Natarajan C M, Tanner M G, Hadfield R H, 2012, Supercond. Sci. Technol. 25, 063001 and the papers in this issue of Supercond. Sci. Technol.

5. Marsili F, et al 2013, Nature Photonics 7, 210

6. Perez de Lara D, Ejrnaes M, Casaburi A, Lisitskiy M, Cristiano R, Pagano S, Gaggero A, Leoni R, Golt'sman G, and. Voronov B, 2008, J. Low Temp. Phys. 151, 771

7. Suzuki K, Miki S, Shiki S, Wang Z, Ohkubo M, 2008 Appl. Phys. Express 1, 031702 and Suzuki K, Miki S, Wang Z, Kobayashi Y, Shiki S, Ohkubo M, 2008 J. Low Temp. Phys. 151, 766

8. Dalton (symbol: Da) is the standard unit that is used for indicating mass on an atomic or molecular scale. The atomic mass unit (symbol: amu) was also used but is technically an obsolete unit based on oxygen, which was replaced in 1961.

9. Twerenbold D, Vuilleumier J L, Gerber D, Tadsen A, Van den Brandt B, Gillevet P M 1996 Appl. Phys. Lett. 68, 3503

10. For a review on sub-kelvin superconducting detectors for TOF-MS see also Frank M, Labov S E, Westmacott G, Benner W H 1999 Mass Spectrometry Reviews 18, 155

11. Hilton G C, Martinis J M, Wollman D A, Irwin K D, Dulcie L L, Gerber D, Gillevet P M, Twerenbold D 1998 Nature 391, 672

12. No information is available about Micromizer nowadays. It was commercialized at the beginning of the last decade by Comet and realized with a collaboration between Comet and Genspec.

13. Casaburi A, Esposito E, Ejrnaes M, Suzuki K, Ohkubo M, Pagano S, Cristiano R, 2012 Supercond. Sci. Technol. 25, 115004

14. Marksteiner M, Divochiy A, Sclafani M, Haslinger P, Ulbricht H, Korneev A, Semenov A, Gol'tsman G, Arndt M 2009 Nanotechnology 20455501

15. Sclafani M, Marksteiner M, McLennan Keir F, Divochiy A, Korneev A, Semenov A, Gol'tsman G, Arndt M, 2012, Nanotechnology 23, 065501

16. Gerlich S, Eibenberger S, Tomandl S, Nimmrichter S, Hornberger K, Fagan P J, Tüxen J, Mayor M, Arndt M (2011) Nature Commun. 2, 263

17. Suzuki K, Shiki S, Ukibe M, Koike M, Miki S, Wang Z, Ohkubo M, 2011 Appl Phys Express 4083101

18. Rosticher M, Ladan F R, Maneval J P, Dorenbos S N, Zijlstra T, Klapwijk T M, Zwiller V, Lupaşcu A, Nogues G, 2010 Appl. Phys. Lett. 97183106

19. Ohkubo M, (2015) International Standards for Superconducting Electronic Devices Superconducting Sensors and Detectors, IEEE/CSC \& ESAS European superconductivity news forum No. 32, ST434

20. Kerman A J, Dauler E A, Keicher W E, Yang J K W, Berggren K K, Gol'tsman G, Voronov B, 2006 Appl. Phys. Lett. 88111116

21. Casaburi A, Ejrnaes M, Zen N, Ohkubo M, Pagano S, and Cristiano R, 2011 Appl. Phys. Lett. 98023702 
22. Ovchinnikov Yu N, Kresin V Z, 1998 Phys. Rev. B 5812416

23. Kozorezov A G, Volkov A F, Wigmore J K, Peacock A, Poelaert A, and den Hartog R, 2000 Phys. Rev. B 6111807

24. E. M. Men'shchikov, I. G. Gogidze, A. V. Sergeev, A. I. Elant'ev, P. B.Kuminov, G. N. Gol'tsman, and E. M. Gershenzon, 1997 Tech. Phys. Lett. 23486

25. Kaplan S B 1979 J. Low Temp. Phys. 37343

26. Ohkubo M, Shiki S, Ukibe M, Tomita S, Hayakawa S 2011 Intern J. Mass Spectrometry 299 94

27. Semenov A, Engel A, Hubers H-W, Il'in K, Siegel M 2005 Eur. Phys. J. B 47495

28. Hofherr M, Rall D, Ilin K, Siegel M, Semenov A, Hubers H-W, Gippius N A, $2010 \mathrm{~J}$. Appl. Phys. 108014507

29. Semenov A D, Gol'tsman G N, Korneev A A, 2001 Physica C 351, 349

30. Renema J J, Frucci G, Zhou Z, Mattioli F, Gaggero A, Leoni R, de Dood M J A, Fiore A, van Exter M P, 2013 Phys. Rev. B 87174526

31. Renema J J, et al 2014 Phys Rev. Letts. 112, 117604

32. Zotova A N and D Y Vodolazov 2012 Phys. Rev. B 85024509

33. Bulaevskii L N, Graf M J, Batista C D, Kogan V G 2011 Phys. Rev. B 83144526

34. Kadin A M, Leung M, Smith A D 1990 Phys. Rev. Lett. 653193

35. Zen N, Shibata H, Mawatari Y, Koike M, and M. Ohkubo M, 2015 Appl. Phys. Lett. 106, 222601

36. Zen N, Chen Y, Suzuki K, Ohkubo M, Miki S, and Wang Z 2009 IEEE Trans. Appl. Super. 19, 354 .

37. Suzuki K, Miki S, Shiki S, Kobayashi Y, Chiba K, Wang Z,. Ohkubo M 2008 Physica C 468 2001

38. Suzuki K, Miki S, Shiki S, Zen N, Wang Z, Ohkubo M 2009 Physica C 469167

39. Koppenaal D W, Barinaga C J, Bonner Denton M, Sperline R P, Hieftje G M, Schilling G D, Andrade F J, Barnes J H 2005 J. Anal. Chem. 77, 418

40. Ohkubo M, et al 2012 J. Low Temp. Phys. 167943

41. Suzuki K, Ohkubo M, Ukibe M, Chiba-Kamoshida K, Shiki S, Miki S, Wang Z 2010 Rapid Commun. Mass Spectrom. 243290

42. Ejrnaes M, Cristiano R, Quaranta O, Pagano S, Gaggero A, Mattioli F, Leoni R, Voronov B, and Gol'tsman G 2007 Appl. Phys. Lett. 91262509

43. Ejrnaes M, Casaburi A, Quaranta O, Marchetti S, Gaggero A, Mattioli F, Leoni R, Pagano S, Cristiano R 2009 Supercond. Sci. Technol. 22055006

44. Marsili F, Najafi F, Dauler E, Bellei F, Hu X, Csete M, Molnar R J, Berggren K K 2011 Nano Lett. 112048 
45. Casaburi A, Zen N, Suzuki K, Ejrnaes M, Pagano S, Cristiano R, Ohkubo M 2009 Appl. Phys. Lett. 94212502

46. Zen N, Casaburi A, Shiki S, Suzuki K, Ejrnaes M, Cristiano R, Ohkubo M 2009 Appl. Phys. Lett. 95172508

47. Ejrnaes M, Casaburi A, Cristiano R, Quaranta O, Marchetti S, Pagano S 2009 J Mod Optics 56, 390

48. Marsili F, Najafi F, Herder C, Berggren K K 2011 Appl. Phys. Lett. 98093507

49. Marsili F, Najafi F, Dauler E, Molnar R J, Berggren K K 2012 Appl. Phys. Lett. 100112601

50. Annunziata A J, et al 2010 J. Appl. Phys. 108084507

51. Kerman A J, Yang J K W, Molnar R J, Dauler E A, Berggren K K 2009 Phys. Rev. B 79 100509(R)

52. Casaburi A, Heath R M, Tanner M G, Cristiano R, Ejrnaes M, Nappi C, Hadfield R H 2013 Appl. Phys. Lett. 103013503

53. Casaburi A, Heath R M , Tanner M G, Cristiano R, Ejrnaes E, Nappi C, Hadfield R H 2014 Supercond. Sci. Technol. 27044029

54. Zen N, Shiki S, Ukibe M, Koike M, Ohkubo M 2014 Appl. Phys. Lett. 104012601

55. Zen N, Fujii G, Shiki S, Ukibe M, Koike M, Ohkubo M 2015 Chin. Phys. B 24 in press

56. Sano K, Muramatsu Y, Yamanashi Y, Yoshikawa N, Zen N, Ohkubo M 2014 Physica C 504 97 


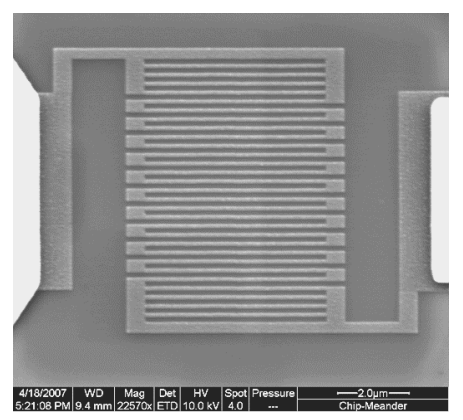

Figure 1. SEM photograph of a superconducting single particle detector (SSPD) with the typical meander geometry.

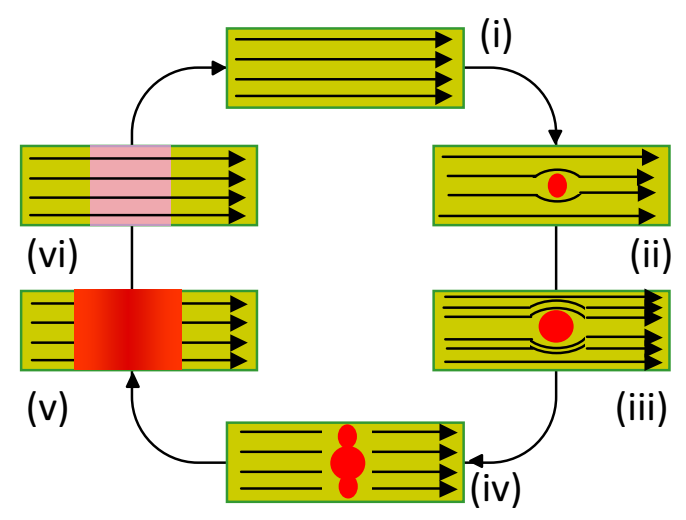

Figure 2. Schematic illustrating the SSPD detection cycle. (i) The superconducting strip maintained well below the critical temperature is direct current (DC) biased just below the critical current. (ii) When a particle/photon is absorbed by the strip a small resistive hotspot is created. (iii) The supercurrent is forced to flow along the periphery of the hotspot. Since the nanowires are narrow, the local current density around the hotspot increases, exceeding the superconducting critical current density. (iv) This in turn leads to the formation of a resistive barrier across the width of the strip. (v) Joule heating (via the DC bias) aids the growth of resistive region along the axis of the strip until the current flow is blocked and the bias current is shunted by the external circuit. (vi) This allows the resistive region to collapse and the strip becomes fully superconducting again. The bias current through the strip returns to the original value (i). (Inspired from figure 1(a) of Ref [4]) 


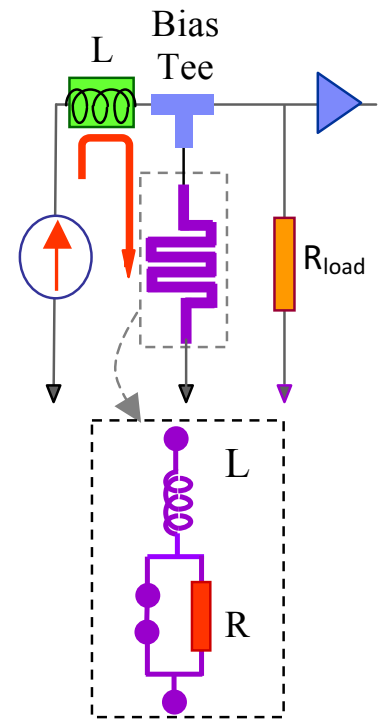

a)

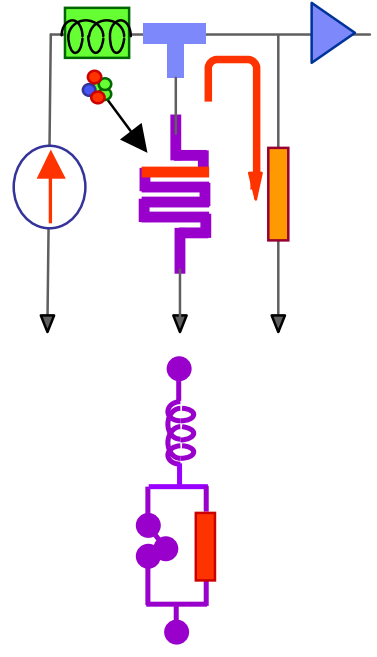

b)

Figure 3. Schematic of the SSPD bias and readout circuit: the SSPD is connected via a bias T to the direct (DC) current supply and the load resistance $R_{\mathrm{L}}$; The electrical equivalent of the SSPD is also shown: the kinetic inductance $L_{\mathrm{k}}$ in series with the block constituted by the parallel of the resistance $R_{\mathrm{n}}$ and the switch. a) In the superconducting state, the current flows in the meander: the switch is closed and shunts $R_{\mathrm{n}}$. b) The arrival of a particle switches the SSPD into the normal state and the current is diverted into the load $R_{\mathrm{L}}$; the output voltage across $R_{\mathrm{L}}$ is readout by the roomtemperature amplifier; in the electrical equivalent, the switch opens and adds a resistance $R_{\mathrm{n}}$ in series. 
(a)

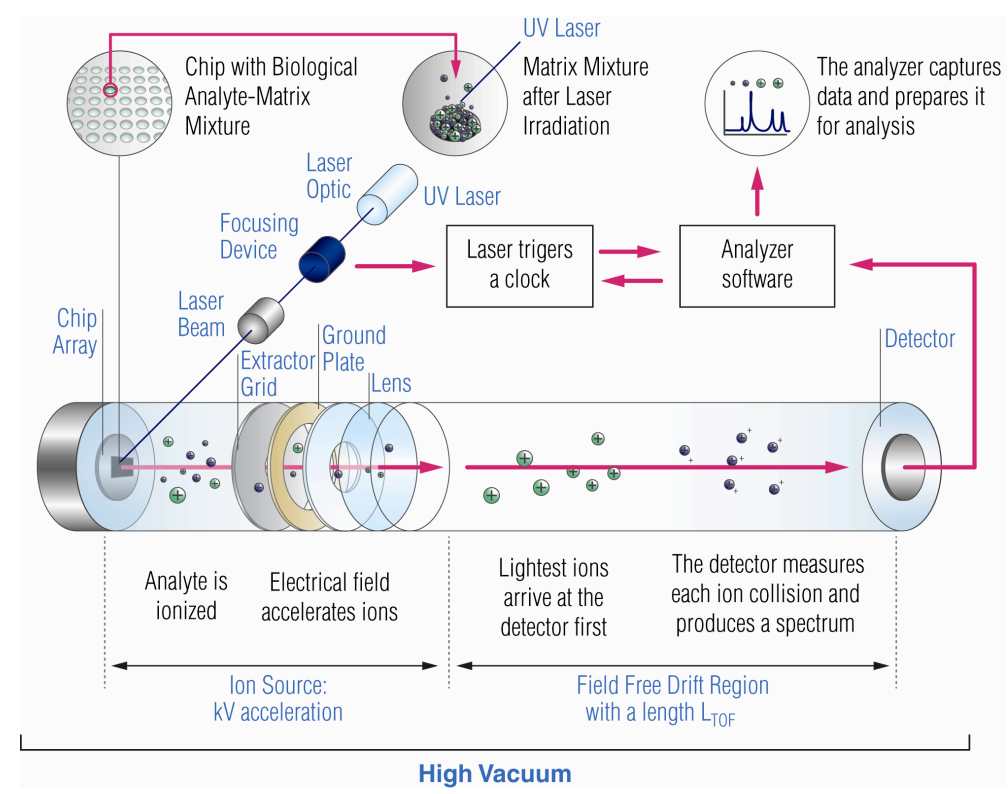

(b)

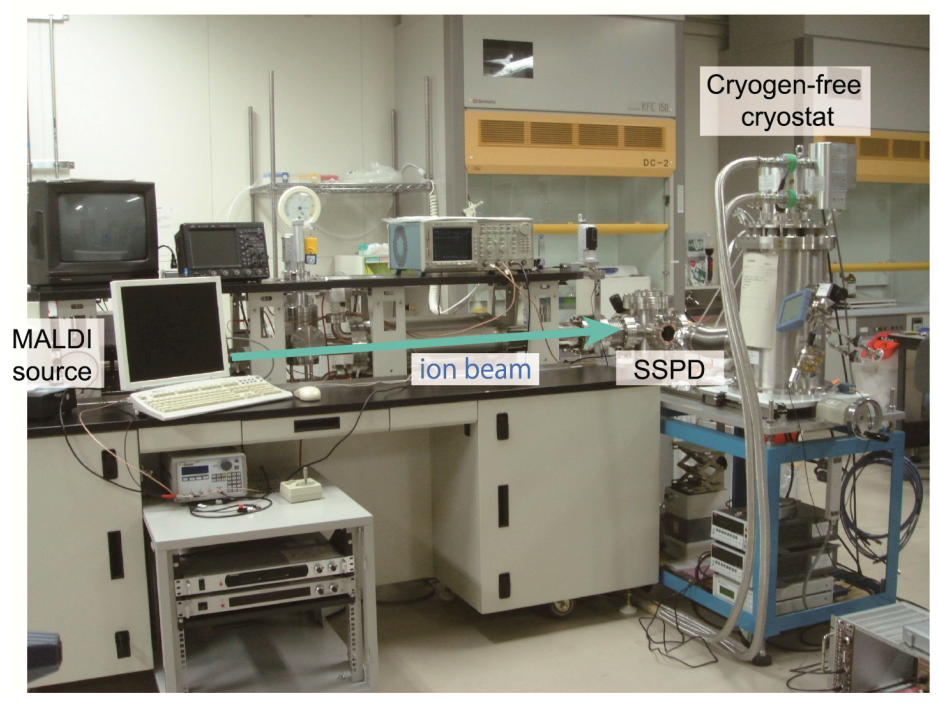

Figure 4. (a) Illustration of the operation mode of a time-of-flight mass spectrometer with a Matrix Assisted Laser Desorption/Ionization (MALDI) source. (b) MALDI-TOF MS with a SSPD realized in a practical instrument at AIST in Tsukuba, Japan. The cryogen-free cryostat is added to a commercial TOF-MS instrument to cool down the SSPD. The picture shows the setup for the linear mode. 


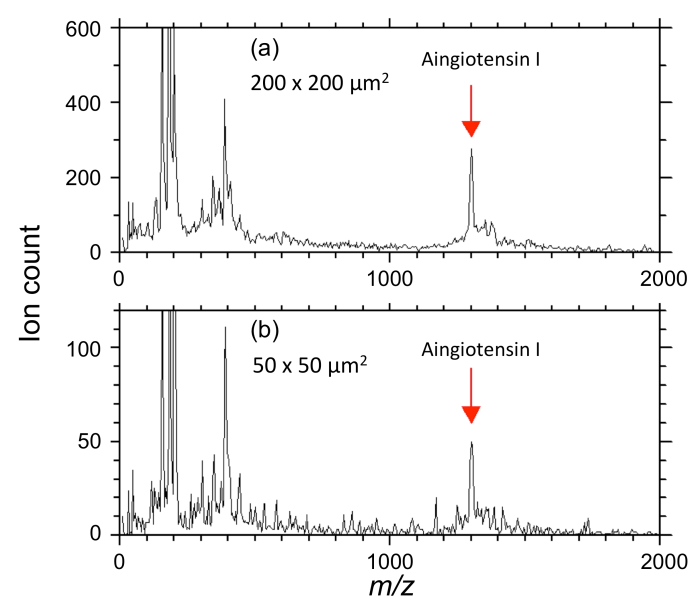

Figure 5. Mass spectra of Angiotensin I obtained by the NbN-SSPD with the sensor size of (a) $200 \times 200 \mu \mathrm{m}^{2}$ and (b) $50 \times 50 \mu \mathrm{m}^{2}$ (after [38]). The spectra were acquired over 10,000 laser shots.

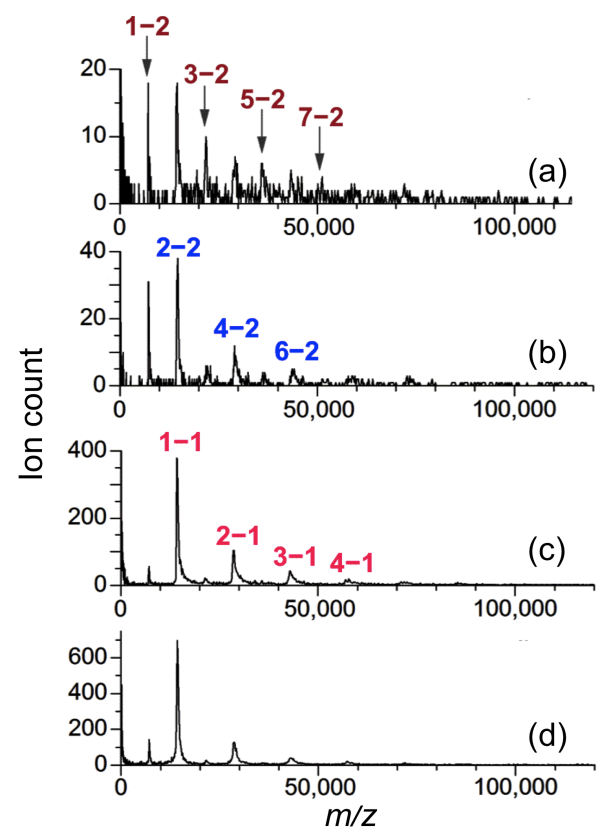

Figure 6. $\mathrm{m} / \mathrm{z}$ spectra of the lysozyme multimers by the SSPD. Full spectra at different bias points in $I / I_{\mathrm{c}}: 0.60$ (a), 0.65 (b), 0.70 (c) and 0.80 (d). The number labelling (multimerization numbercharge number) shows the peak positions for the monomer and multimer ions: for example, 2-1 for the singly charged dimer. (after [41]). 


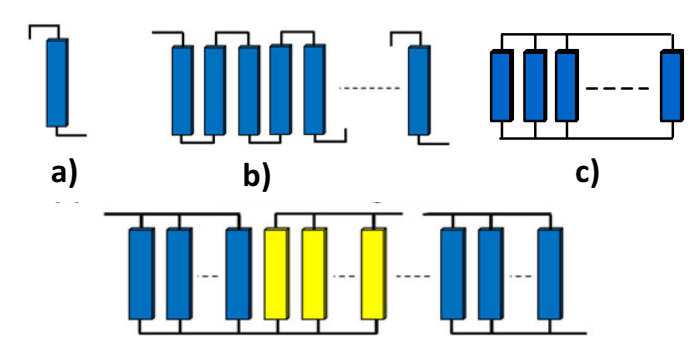

d)

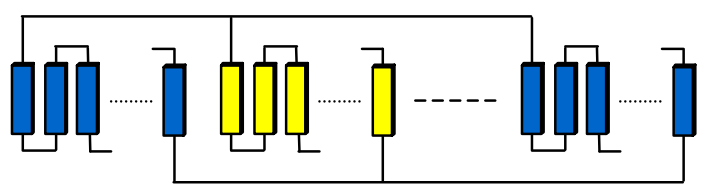

e)

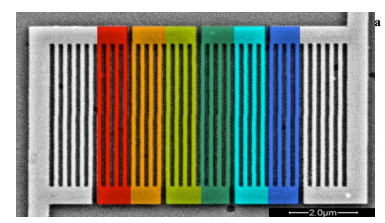

f)

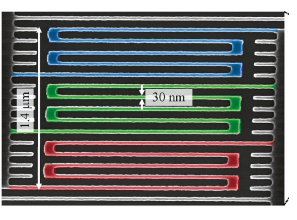

g)

Figure 7. Sketch of the SSPD configurations: (a) single-strip; (b) pure meander; and (c) pure parallel (Type-0) configuration; (d) Type-I configuration where blocks of parallel strips (different colours) form a meander; (e) Type-II configuration where meanders (different colours) are connected in parallel; (f) SEM photo with false colours of a Type I configuration: the blocks of parallel strips are distinguished by different colors; g) SEM photo (after [44]) with false colours of a Type II configuration ( the meanders have different colours). 

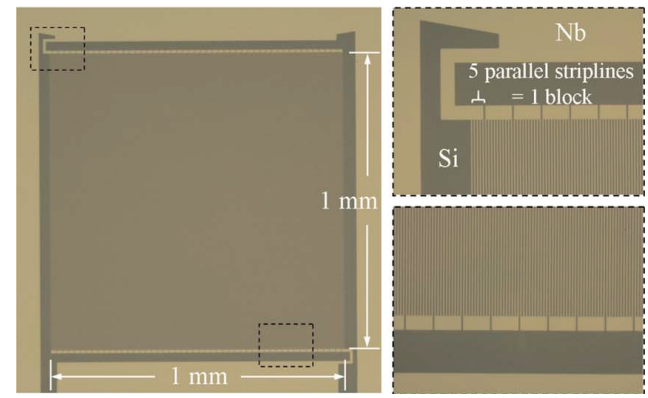

Figure 8. Optical micrograph of the 1-mm-square, five parallel $\mathrm{Nb}$-SSPD. The parts surrounded by the dashed squares are enlarged in the right side (after [46]).

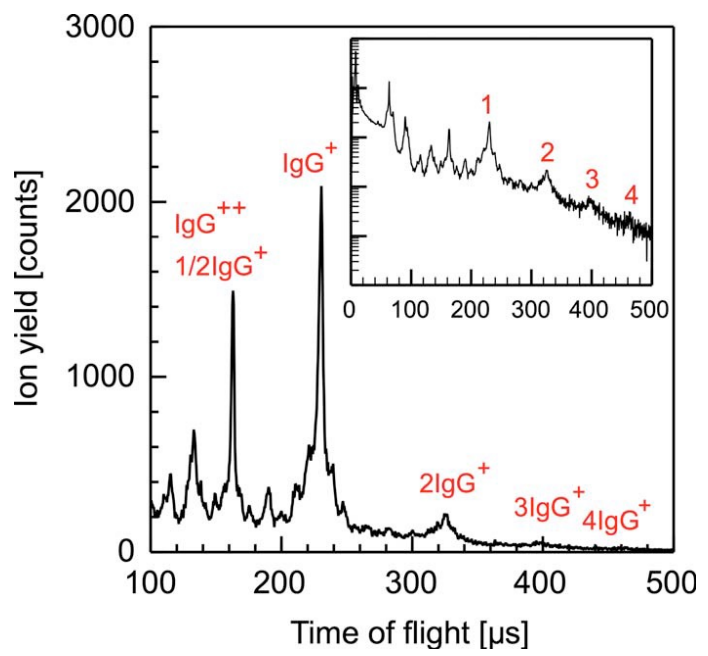

Figure 9. Time-of-flight spectrum of $\operatorname{IgG}(146 \mathrm{kDa})$ multimers obtained by the SSPD in figure 8 . The inset shows the logarithmic plot with the numbers of oligomerization. (After [46]). 


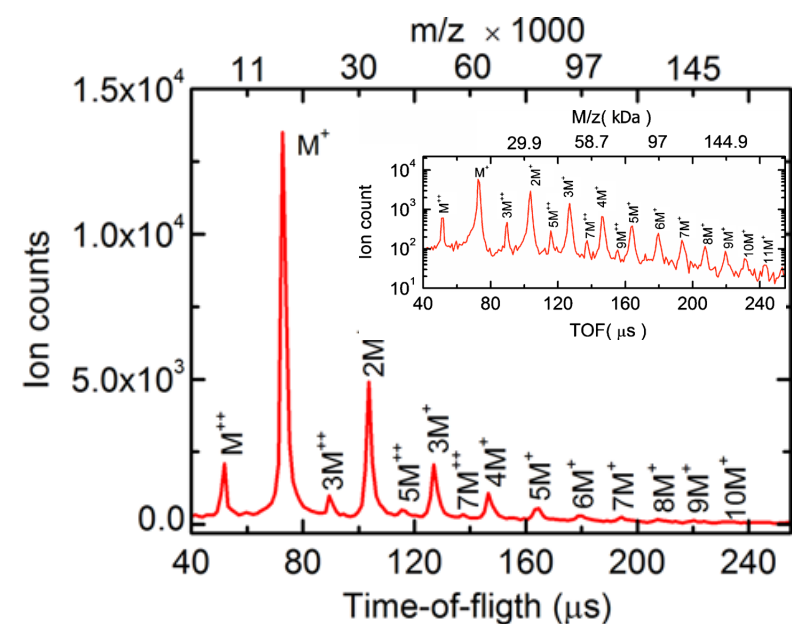

Figure 10. TOF spectrum for lysozyme molecules (mass weight $14.3 \mathrm{kDa}$ corresponding to a TOF $=73.6 \mu \mathrm{s})$ of the $2 \times 2 \mathrm{~mm}^{2}$ parallel-SSPD acquired by operating the detector at a bias current of $0.53 I_{\mathrm{c}}$ and a temperature of $4.2 \mathrm{~K}$. The peaks are labelled with the symbol $\mathrm{M}+$ and so on that indicate the multimer base of the molecule complex and the corresponding ionization charge. The inset shows the same spectrum where the ion counts are plotted on a logarithmic scale to better show the very high signal to background ratio of the peaks.
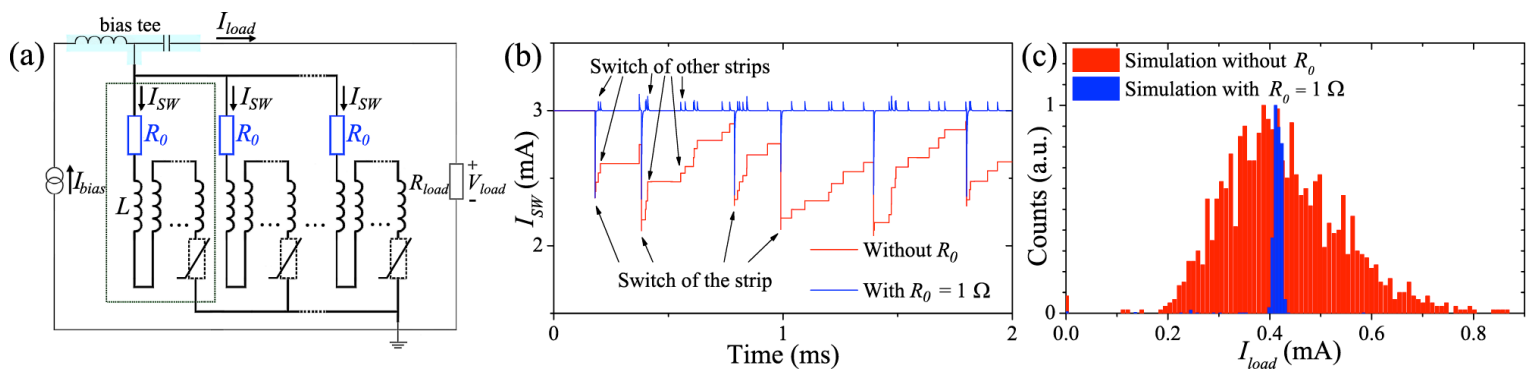

Figure 11. Simulation of output pulse height distribution for Type-II configuration. The equivalent circuit is shown in (a), in which ten parallel strips switch to normal state independently and randomly, simulating an actual ion impact. The simulation was conducted with and without the resistors $R_{0}=1 \Omega$. A bias current $I_{\text {bias }}=30 \mathrm{~mA}$ was assumed. Time evolution of $I_{S W}$ in a strip with (blue) and without (red) $R_{0}$ is shown in (b). Corresponding histograms of $I_{\text {load }}$ are plotted in (c). (after [54]). 


\begin{tabular}{lccccc}
\hline \hline Applications & $\begin{array}{c}\text { Energy } \\
(\mathrm{keV})\end{array}$ & Material & $\begin{array}{c}w \\
(\mathrm{~nm})\end{array}$ & $\begin{array}{c}d \\
(\mathrm{~nm})\end{array}$ & $\begin{array}{c}\text { Area } \\
(\mu \mathrm{m} \times \mu \mathrm{m})\end{array}$ \\
\hline Angiotensin I ions [36] & $\sim 20$ & $\mathrm{Nb}$ & $200-300$ & 40 & $200 \times 200$ \\
Ar ions [17] & $0.5-9.0$ & $\mathrm{NbN}$ & 800 & 10 & $200 \times 200$ \\
$\mathrm{He}^{+}$ions [15] & $0.2-1.0$ & $\mathrm{NbN}$ & 100 & 4 & $20 \times 20$ \\
slow neutrals molecules [14] & $\geq 10^{-4}$ & $\mathrm{NbN}$ & 100 & 4 & $20 \times 20$ \\
electrons [18] & $\sim 1$ & $\mathrm{NbTiN}$ & 100 & 6 & $10 \times 10$ \\
\hline \hline
\end{tabular}

Table I. Comparison of meander-SSPDs used in different applications which imply different types of particles and values of energy. The size of the detectors is also reported.

\begin{tabular}{ccccccc}
\hline \hline SSPD & Material & $\begin{array}{c}\text { Area } \\
(\mu \mathrm{m} \times \mu \mathrm{m})\end{array}$ & $\begin{array}{c}w \\
(\mathrm{~nm})\end{array}$ & $\begin{array}{c}d \\
(\mathrm{~nm})\end{array}$ & $\begin{array}{c}\tau_{\mathrm{r}} \\
(\mathrm{ps})\end{array}$ & $\begin{array}{c}\tau_{\mathrm{d}} \\
(\mathrm{ns})\end{array}$ \\
\hline \multirow{2}{*}{ Meander [7] } & $\mathrm{NbN}$ & $50 \times 50$ & 200 & 7 & 640 & 22 \\
Meander [38] & $\mathrm{NbN}$ & $200 \times 200$ & 800 & 10 & 900 & $\geq 20$ \\
Parallel [13] & $\mathrm{NbN}$ & $2000 \times 2000$ & 1000 & 50 & 500 & 2.3 \\
\hline \hline
\end{tabular}

Table II. Rise- and decay-time of meander-SSPDs with increasing areas. The last line reports data of a parallel-SSPD for comparison. 